\section{(6) \\ OPEN ACCESS}

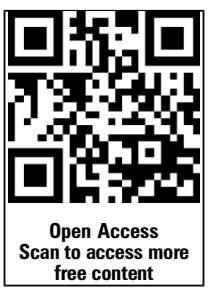

- Additional material is published online only. To view this file please visit the journal online (http://dx.doi.org/10. 1136/thoraxjnl-2015-208050)

\section{${ }^{1} \mathrm{MRC}$ Centre for Inflammation Research, University of Edinburgh, Edinburgh, UK ${ }^{2}$ Clinical Microbiology, NHS Lothian, Edinburgh, UK ${ }^{3}$ Institute of Cellular Medicine, Newcastle University, Newcastle, UK \\ ${ }^{4}$ Centre for Infection and Immunity, Queen's University, Belfast, UK \\ ${ }^{5}$ Department of Microbiology, Belfast Health \& Social Care \\ Trust, Belfast, UK \\ ${ }^{6}$ Division of Anaesthesia, Department of Medicine, University of Cambridge, Cambridge, UK}

\section{Correspondence to} Dr A Conway Morris, Division of Anaesthesia, University of Cambridge, Box 93 Addenbrooke's Hospital, Hills Road, Cambridge CB2 2QQ, UK; mozza@doctors.org.uk

TJN and NJG contributed equally.

ACM and AJS indicate joint senior authorship of this work.

Received 10 November 2015 Revised 12 January 2016 Accepted 4 February 2016 Published Online First 12 April 2016

\section{CrossMark}

To cite: Nolan TJ, Gadsby NJ, Hellyer TP, et al. Thorax 2016;71:594-600.

\title{
Low-pathogenicity Mycoplasma spp. alter human monocyte and macrophage function and are highly prevalent among patients with ventilator-acquired pneumonia
}

\author{
T J Nolan, ${ }^{1}$ N J Gadsby, ${ }^{2}$ T P Hellyer, ${ }^{3}$ K E Templeton, ${ }^{2}$ R McMullan, ${ }^{4}$ J P McKenna, ${ }^{5}$ \\ J Rennie, ${ }^{1}$ C T Robb, ${ }^{1}$ T S Walsh, ${ }^{1}$ A G Rossi, ${ }^{1}$ A Conway Morris, ${ }^{1,6}$ A J Simpson ${ }^{3}$
}

ABSTRACT

Background Ventilator-acquired pneumonia (VAP) remains a significant problem within intensive care units (ICUs). There is a growing recognition of the impact of critical-illness-induced immunoparesis on the pathogenesis of VAP, but the mechanisms remain incompletely understood. We hypothesised that, because of limitations in their routine detection

Mycoplasmataceae are more prevalent among patients with VAP than previously recognised, and that these organisms potentially impair immune cell function.

Methods and setting 159 patients were recruited from 12 UK ICUs. All patients had suspected VAP and underwent bronchoscopy and bronchoalveolar lavage (BAL). VAP was defined as growth of organisms at $>10^{4}$ colony forming units per ml of BAL fluid on conventional culture. Samples were tested for Mycoplasmataceae (Mycoplasma and Ureaplasma spp.) by PCR, and positive samples underwent sequencing for speciation. 36 healthy donors underwent BAL for comparison. Additionally, healthy donor monocytes and macrophages were exposed to Mycoplasma salivarium and their ability to respond to lipopolysaccharide and undertake phagocytosis was assessed.

Results Mycoplasmataceae were found in 49\% $(95 \% \mathrm{Cl}$ $33 \%$ to $65 \%$ ) of patients with VAP, compared with $14 \%$ (95\% Cl 9\% to $25 \%$ ) of patients without VAP. Patients with sterile BAL fluid had a similar prevalence to healthy donor BAL fluid ( $10 \%(95 \% \mathrm{Cl} 4 \%$ to $20 \%$ ) vs $8 \%(95 \%$ Cl $2 \%$ to $22 \%))$. The most common organism identified was $M$. salivarium. Blood monocytes from healthy volunteers incubated with $M$. salivarium displayed an impaired TNF- $\alpha$ response to lipopolysaccharide $(p=0.0003)$, as did monocyte-derived macrophages (MDMs) $(p=0.024)$. MDM exposed to $M$. salivarium demonstrated impaired phagocytosis ( $p=0.005$ ).

Discussion and conclusions This study demonstrates a high prevalence of Mycoplasmataceae among patients with VAP, with a markedly lower prevalence among patients with suspected VAP in whom subsequent cultures refuted the diagnosis. The most common organism found, $M$. salivarium, is able to alter the functions of key immune cells. Mycoplasmataceae may contribute to VAP pathogenesis.

\section{INTRODUCTION}

Ventilator-acquired pneumonia (VAP) remains a significant cause of morbidity and mortality in

\section{Key messages}

What is the key question?

- What is the prevalence of Mycoplasmataceae among patients with ventilator-acquired pneumonia (VAP), and does this have any pathophysiological relevance?

What is the bottom line?

- Patients with VAP have a high prevalence of Mycoplasmataceae compared with similar patients without VAP, and the most common species found can impair immune cell function.

\section{Why read on?}

- These findings provide a novel insight into the biology of VAP and suggest new potential prevention strategies.

patients admitted to the intensive care unit (ICU). ${ }^{1}$ There is a growing recognition of failure of immune cell function (immunoparesis) in the lung ${ }^{2}$ and peripherally ${ }^{3}{ }^{4}$ in the pathogenesis of VAP. The mediators of this immunoparesis remain incompletely understood, although it is likely that it arises from multifactorial insults involving both host and microbial factors. It is now widely recognised that the predominant route for infection is via 'microaspiration' of organisms from the hypopharynx, allowing colonisation and subsequent infection of the lower airways. ${ }^{1}$

The organisms which cause VAP have traditionally been thought to be conventional bacterial species, such as Staphylococcus aureus and Pseudomonas aeruginosa. ${ }^{5}$ However our understanding of the microbiology of VAP is heavily influenced by the relative ease of microbial culture. In recent years there has been a greater understanding of the human microbiome and the influence of bacteria that are not cultured. ${ }^{6}$ Recent reports have suggested that non-classical organisms, such as Mycoplasma spp., may be present in patients with VAP. $^{7}{ }^{8}$ The methods of detection used in these studies have been variable and there was little evidence of the impact these species may have on host immunity. 
Mycoplasmataceae are members of the mollicutes class of bacteria, which lack a cell wall and have the smallest genomes found in self-replicating organisms. ${ }^{9}$ Their limited biosynthetic capability makes culture and detection problematic, needing specialised growth media and prolonged incubation or reliance on indirect methods such as serology. ${ }^{9}$ A range of Mycoplasmataceae such as Mycoplasma salivarium and M. orale are generally considered to be human commensals, ${ }^{10}$ while others such as M. pneumoniae and Ureaplasma urealyticum are recognised pathogens of the respiratory and genitourinary tract, respectively. However, even the Mycoplasma spp. commonly thought to be commensals have been shown to cause infections in immunocompromised hosts. ${ }^{11}$ Additionally, co-infection with Mycoplasma spp. has been implicated in accelerated progression of HIV/AIDS. ${ }^{12}$

We hypothesised that patients with confirmed VAP would have a higher prevalence of Mycoplasmataceae than patients without VAP, and that the dominant organism found (M. salivarium) would impair the ability of monocytes and macrophages to respond to further bacterial stimuli. Accordingly, this study had two aims; first, to quantify the prevalence of Mycoplasmataceae in two cohorts of patients with suspected VAP and determine which species were present. The second aim was to examine the effect of a classically non-pathogenic Mycoplasma, M. salivarium, on the ability of monocytes and macrophages to respond to pathogenic stimuli.

\section{METHODS}

\section{Patients and volunteers}

We constructed the subject cohorts for this study from clinical samples and data from two recent patient studies and a healthy volunteer cohort. ${ }^{13} 14$ The first patient cohort was recruited from two general, teaching hospital ICUs from 2005 to 2009. Patients were included if they fulfilled clinical criteria for suspected VAP, that is, mechanical ventilation for $>48 \mathrm{~h}$, new infiltrates on chest radiograph, and at least two of the following: temperature $>38^{\circ} \mathrm{C}$, leucocyte count $>11 \times 10^{9}$ per litre of peripheral blood or purulent tracheal secretions.

The second patient cohort was recruited from 12 ICUs across the UK from 2012 to 2013, and met similar enrolment criteria to those in patient cohort 1 . Both cohorts 1 and 2 underwent a standardised bronchoscopy and bronchoalveolar lavage (BAL) as described. ${ }^{13} 14$ In both cohorts VAP was confirmed by growth of organisms at $>10^{4}$ colony forming units per $\mathrm{ml}$ of BAL fluid $(\mathrm{CFU} / \mathrm{mL})$ on conventional culture.

The healthy volunteers were recruited from the University of Edinburgh and from a primary care practice. Volunteers underwent either bronchoscopy and BAL using the same protocol as used in patient cohort 1 , or phlebotomy for extraction of peripheral blood leucocytes for the leucocyte function experiments detailed below.

\section{Sample processing}

BAL fluid was prepared as described, ${ }^{13}$ with the cell-free supernatant being stored at $-80^{\circ} \mathrm{C}$. Aliquots of $\mathrm{BAL}$ fluid from patient cohort 1 and healthy volunteers underwent nucleic acid extraction by one of the authors (NJG) using the DNeasy Blood and Tissue kit (Qiagen, Manchester, UK) with pretreatment protocol for Gram-positive bacteria, according to the manufacturer's instructions. Extractions for patient cohort 2 were undertaken by another author (JPM) using the MagNA pure 96 DNA and viral NA small volume kit (Roche, Indianapolis, Indiana, USA) in a geographically separate laboratory. The extraction in two laboratories was undertaken to confirm that the Mycoplasma detection was not occurring due to contamination of consumables in one or other laboratory. Further confirmation came by running the PCR on saline passed through the same extraction columns but without contact with the patient or volunteer BAL fluid samples.

PCR for Mycoplasma spp. was based on the method of van Kuppeveld et $a l^{15}$ targeting the 16S rRNA gene using forward primer general prokaryotic oligonucleotides-1 (GPO-1) and reverse primer mycoplasma genus-specific oligonucleotides (MGSO) and gel electrophoresis for detection. Positive extracts were sequenced on the reverse strand to determine Mycoplasma spp. using the QIAquick PCR purification kit (Qiagen) for purification, and ABI Prism BigDye Terminator and the ABI 3730 instrument (Applied Biosystems, Life Technologies, Paisley, UK) for Sanger sequencing. Ureaplasma spp. real-time PCR was based on an inhouse unpublished method targeting the urease gene using the following oligonucleotides; UreF: ACG WCG TTT CGA TAT TCC AT, UreR: TTC CRT TAA CTA AGC CRT TT and UreP: 6'FAM-TCG TTT TGA ACC AGG AGA YAA AA-BHQ1. Reactions comprised HotStarTaq (Qiagen) reagents, $0.5 \mu \mathrm{M}$ primer Ure-F, $0.65 \mu \mathrm{M}$ primer UreR, $0.2 \mu \mathrm{M}$ probe UreP and $10 \mu \mathrm{L}$ nucleic acid extract to a final volume of $25 \mu \mathrm{L}$. Real-time PCR was carried out on the ABI 7500 instrument (Applied Biosystems). Ureaplasma spp. positive extracts were speciated using the method of Kong et al ${ }^{16}$ targeting the $5^{\prime}$ end of the MBA gene with primers UMS-57c and UMA222 for U. parvum and UMS-170c and UMA263 for U. urealyticum, followed by gel-based detection.

\section{Human inflammatory cell/Mycoplasma interactions}

Human mononuclear cells were obtained from whole blood from healthy donors using previously described methods. ${ }^{17} 18$ Briefly, leucocytes were obtained by dextran sedimentation, with separation of mononuclear and granulocytic cells over Percoll gradients. CD14+ve monocytes were extracted from the mononuclear fraction using a magnetic bead system (MACS, Miltenyi Biotec, Bisley, UK), and $>95 \%$ purity confirmed by flow cytometry. Monocytes were either used fresh at 300000 per well, or matured into monocyte-derived macrophages (MDMs). MDMs were produced by adhering 300000 monocytes to 24-well tissue culture plates (Corning Costar, Sigma-Aldrich, Gillingham, UK) and incubating in Iscove's modified Dulbecco's medium and $10 \%$ autologous serum for 7 days. MDM maturation was confirmed morphologically using light microscopy.

\section{Exposure to Mycoplasma}

Monocytes and MDMs were initially exposed to three titres of live M. salivarium strain NC10113 (Mycoplasma Experience, Bletchingley, UK), namely multiplicity of infection (MOI) of 1:10 (low), 1:1 (moderate) and 10:1 (high) for $24 \mathrm{~h}$ at $37^{\circ} \mathrm{C}$ and $5 \% \mathrm{CO}_{2}$. Cell death was assessed by nuclear staining using Sytox Green (Life Technologies) and fluorescence microscopy, counting the number of fluorescent cells in three randomly selected fields per well, with a minimum of 100 cells per field. All experiments were conducted in duplicate using a minimum of $\mathrm{n}=3$ individual donors.

\section{Influence of Mycoplasma on response to lipopolysaccharide} Monocytes and MDMs were cultured as above and exposed to either live M. salivarium or vehicle control for $24 \mathrm{~h}$. Supernatants were removed, centrifuged at $200 \mathrm{~g}$ for $10 \mathrm{~min}$ and frozen for later analysis. In subsequent experiments monocytes and MDMs were cultured with $M$. salivarium or vehicle control for $24 \mathrm{~h}$ and then, without washing or changing supernatant, exposed to lipopolysaccharide (Escherichia coli 0127:B8, 
Sigma-Aldrich, Gillingham, UK) at $100 \mathrm{ng} / \mathrm{mL}$ for a further $24 \mathrm{~h}$, before supernatants were removed and processed as above. Cytokines were assayed using Cytometric Bead Array (BD Biosciences, Oxford, UK).

\section{Effect of Mycoplasma on phagocytosis by MDMs}

MDMs were exposed to live M. salivarium or vehicle control for either short $(60 \mathrm{~min})$ or long $(24 \mathrm{~h})$ incubations. Following this the cells were presented with a phagocytic target, pHrodo Red Zymosan (Life Technologies) that had been opsonised in autologous donor serum. Cells were exposed to $0.02 \mathrm{mg} / \mathrm{mL}$ zymosan for $30 \mathrm{~min}$ before being placed on ice and phagocytosis assessed by fluorescence microscopy.

\section{Statistical analysis}

Categorical variables were expressed as proportions and compared using Fisher's exact test. Continuous variables were expressed as mean values and compared using one-way analysis of variance (ANOVA) with Bonferroni's post hoc test for comparison between individual conditions. All analyses were performed using Prism (Graphpad Software, La Jolla, California, USA). $\mathrm{p} \leq 0.05$ was considered statistically significant.

\section{Ethical approval}

The samples from patient cohorts 1 and 2 were collected in studies approved by the Lothian Research Ethics Committee (REC) (LREC/2002/8/19) and NRES North East REC (11/NE/ 0242) and Scotland A REC (11/SS/0089), respectively, with the informed consent/assent from the next of kin. The healthy volunteer BAL fluid was obtained from volunteers in a study approved by Lothian REC (06/S1101/50) with all volunteers giving written informed consent. The monocyte and MDM experiments were conducted using cells obtained under the authorisation of the Lothian REC (08/S1103/38) following written, informed consent.

\section{RESULTS}

\section{Settings and patients}

In total there were 159 patients (67 from cohort 1, 92 from cohort 2) for analysis, of whom 43 (13 from cohort 1 and 30 from cohort 2) had confirmed VAP. The demographics of patient cohorts 1 and 2 have been described previously; ${ }^{13} 14$ briefly the patients were $69 \%$ male, with a median age of 60 years (IQR 48-71), 45\% were admitted to ICU with surgical

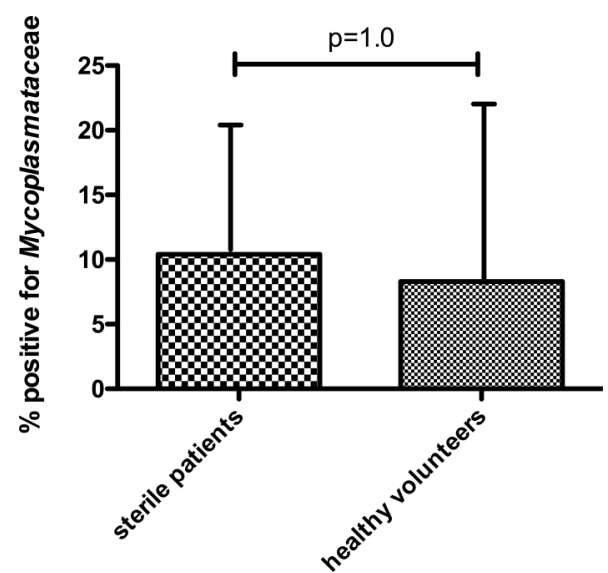

Figure 1 Proportion of healthy volunteers and patients with no microbial growth (by conventional culture) in whom Mycoplasmataceae were detected. Data shown as proportion and upper $95 \% \mathrm{Cl}$. p Value by Fisher's exact test.

diagnoses with the remainder admitted with medical diagnoses. Median APACHE II score was 20 (IQR 16-24), ICU mortality was $28 \%$ and hospital mortality $35 \%$. Further demographic details are shown in the online supplementary table S1. Thirty-six healthy donors underwent BAL using the same protocol as cohort $1 .^{13}$

\section{Detection of Mycoplasma and Ureaplasma spp.}

Detection of Mycoplasmataceae (Mycoplasma and Ureaplasma spp.) occurred significantly more frequently in patients with VAP compared with those without VAP (table 1). This was the case for both patient cohorts. Among patients without VAP, the frequency of Mycoplasmataceae was higher in those who had some growth on culture as opposed to those whose BAL fluid was sterile by conventional culture, although none of these differences reached statistical significance (table 1). The frequency of Mycoplasmataceae detection did not differ significantly between patients with sterile cultures and healthy volunteers $(p=1.0$ by Fisher's exact test, figure 1$)$, nor was the trend in difference between all non-VAP patients and healthy volunteers significant (14\% (95\% CI $9 \%$ to $25 \%)$ vs $8 \%$ (95\% CI $2 \%$ to $22 \%), p=0.57$ by Fisher's exact test).

The majority (82\%) of Mycoplasmataceae detected in patients were Mycoplasma spp., and within this group M. salivarium

Table 1 Frequency of Mycoplasmataceae detection in patients with and without VAP confirmed by conventional microbiological culture




Table 2 Species of atypical organism detected in patients (two patients had both Mycoplasma spp. and Ureaplasma spp. detected)

\section{Organism}

Mycoplasma hominis

Mycoplasma salivarium

Ureaplasma parvum

Ureaplasma urealyticum

Unable to speciate (Ureaplasma sp +ve by PCR)
Mixed species of Mycoplasma, unable to further speciate n/frequency

$2(5 \%)$

$22(56 \%)$

$1(3 \%)$

$4(10 \%)$

$8(21 \%)$

$2(5 \%)$ was the dominant organism detected (table 2). Two healthy volunteers were positive for Mycoplasma spp. by PCR but sequencing revealed non-Mycoplasmataceae organisms (Solobacterium moorei) and these samples were excluded from the 'positive' samples. Among the remaining three healthy volunteer samples that were positive for Mycoplasma spp. sequencing revealed one $M$. salivarium and two mixed species.

The presence of atypical organisms did not differ significantly between patients with VAP arising from differing aetiological classes (table 3).

\section{Effect of $M$. salivarium coculture on monocyte and MDM function}

Following the discovery of a high prevalence of $M$. salivarium among patients with VAP (52\% of Mycoplasmataceae in patients with VAP were M. salivarium), we hypothesised that this species may have a role to play in the pathogenesis of VAP. We therefore examined the effect of $M$. salivarium infection on the ability of key immune cells, monocytes and macrophages, to respond to bacterial stimuli.

\section{M. salivarium and cellular toxicity}

Neither monocytes nor MDMs exposed to live M. salivarium for $24 \mathrm{~h}$ at MOIs of 1:10 (low) and 1:1 (moderate) exhibited significantly greater cell death than untreated cells (figure 2A, B). A high MOI (10:1) induced 30\% cell death in MDMs (figure 2B, far right column). Monocytes appeared relatively resistant to such effects (figure 2A), although demonstrated an overall higher basal level of cellular death than MDMs. Subsequent experiments were confined to low and moderate exposures for both cell types.

M. salivarium selectively impairs the ability of immune cells to respond to stimulation with lipopolysaccharide

As expected, lipopolysaccharide (LPS) alone significantly increased the secretion of tumour necrosis factor (TNF)- $\alpha$,

Table 3 Mycoplasmataceae detection among patients with VAP (ie, growth at $>10^{4} \mathrm{CFU} / \mathrm{mL}$ ) of various aetiologies

\begin{tabular}{|c|c|c|}
\hline VAP pathogen & $\mathrm{N}=$ & $\begin{array}{l}\text { Proportion positive for atypical organisms, \% } \\
\text { (n) }\end{array}$ \\
\hline Gram-positive bacteria & 12 & $58(7)$ \\
\hline $\begin{array}{l}\text { Gram-negative } \\
\text { bacteria }\end{array}$ & 20 & $35(7)$ \\
\hline Fungi and yeasts & 7 & $57(4)$ \\
\hline Mixed organism types & 4 & $75(3)$ \\
\hline
\end{tabular}

interleukin (IL) 6 and IL-10 from monocytes and MDM (figure 3 ); in general $M$. salivarium alone had a similar but smaller effect (see online supplementary tables S3 and S4). However, the TNF- $\alpha$ response to an LPS challenge was impaired following both low and moderate $M$. salivarium exposure in monocytes and macrophages $(p=0.0003$ and $p=0.0024$ by ANOVA, respectively) (figure $3 \mathrm{~A}, \mathrm{~B}$ ). A similar although slightly less pronounced pattern was seen with IL-6 $(p=0.0006$ and $p=0.04)$ and IL-10 $(\mathrm{p}=0.02$ and $\mathrm{p}=0.004)$ secretion (figure $3 \mathrm{C}-\mathrm{F})$. IL-8 secretion showed a contrasting pattern, being unchanged in monocytes $(p=0.55)$ while macrophages showed enhanced IL-8 secretion with Mycoplasma exposure ( $\mathrm{p}=0.0013$ ) (figure 3G, H).

\section{M. salivarium impairs phagocytosis by macrophages}

Phagocytosis of microbes and other particulate matter by macrophages is a key part of pulmonary defences, as it directs destruction of the microorganism and facilitates antigen presentation to effector cells ${ }^{19}$ M. salivarium significantly impaired phagocytosis by macrophages following $24 \mathrm{~h}$ exposure (figure 4), with
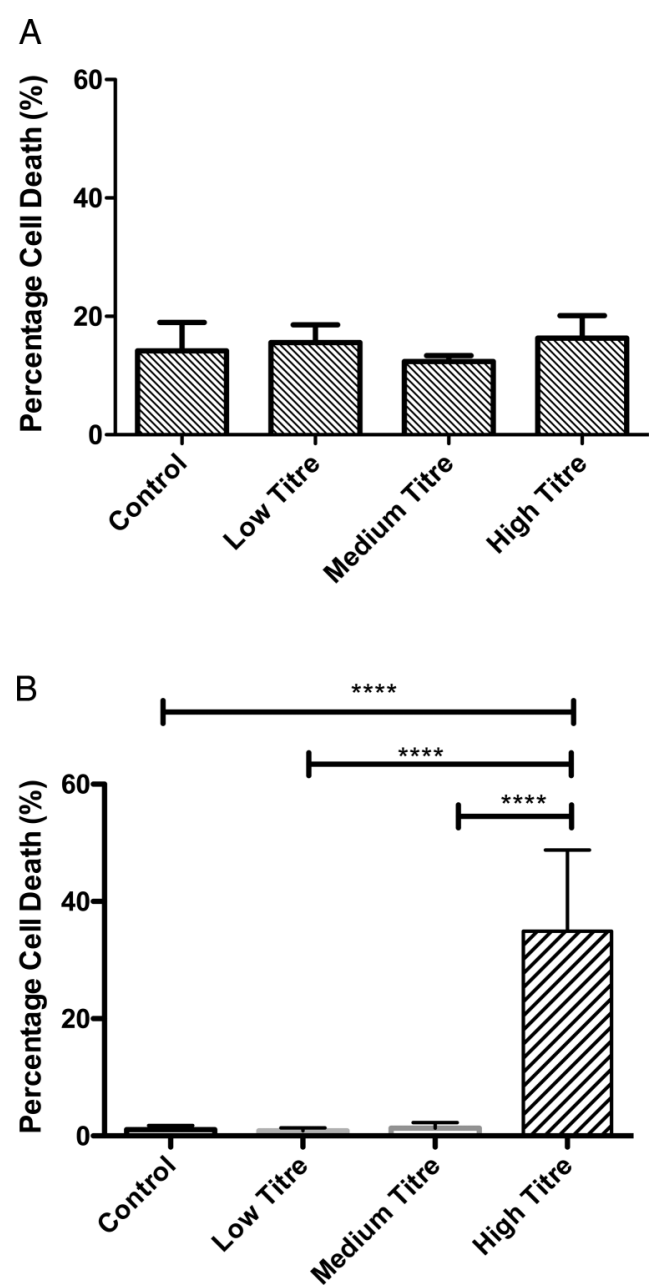

Figure 2 Cell death associated with Mycoplasma salivarium in monocytes and monocyte-derived macrophages (MDMs). (A). Numbers of Sytox green positive monocytes as a proportion of total cells from three randomly selected fields per condition, $n=3$ individual donors. $p=0.56$ by ANOVA. Data shown as mean and SD. (B). Numbers of Sytox green positive MDMs as a proportion of total cells from three randomly selected fields per condition, $n=3$ individual donors. $\mathrm{p}<0.0001$ by ANOVA, ${ }^{* * * *} \mathrm{p}<0.0001$ by Bonferroni's post hoc test, all other comparisons non-significant by Bonferroni's test. Data shown as mean and SD. 
Figure 3 Effect of exposing monocytes and monocyte-derived macrophages (MDMs) to Mycoplasma salivarium and lipopolysaccharide (LPS), on cytokine secretion. (A) TNF- $\alpha$ secretion by monocytes, $p=0.0003$ by ANOVA. (B) TNF- $\alpha$ secretion by MDMs, $\mathrm{p}=0.0024$ by ANOVA. (C) Interleukin (IL) 6 secretion by monocytes, $\mathrm{p}=0.0006$ by ANOVA. (D) IL- 6 secretion by MDMs, $p=0.04$ by ANOVA. (E) IL-10 secretion by monocytes, $p=0.02$ by ANOVA. (F) IL-10 secretion by MDMs, $p=0.004$ by ANOVA. (G) IL-8 secretion by monocytes, $p=0.55$ by ANOVA. $(H)$ IL-8 secretion by MDMs, $p=0.0013$ by ANOVA. ${ }^{*} p<0.05,{ }^{* *} p<0.01$, ${ }^{* * *} p<0.001$ by Bonferroni's post-hoc test, all other comparisons were non-significant by Bonferroni's test. Results are from duplicates of $n=3$ individual healthy donors, with 300000 cells per well. Data shown as mean and SD. MOI, multiplicity of infection.
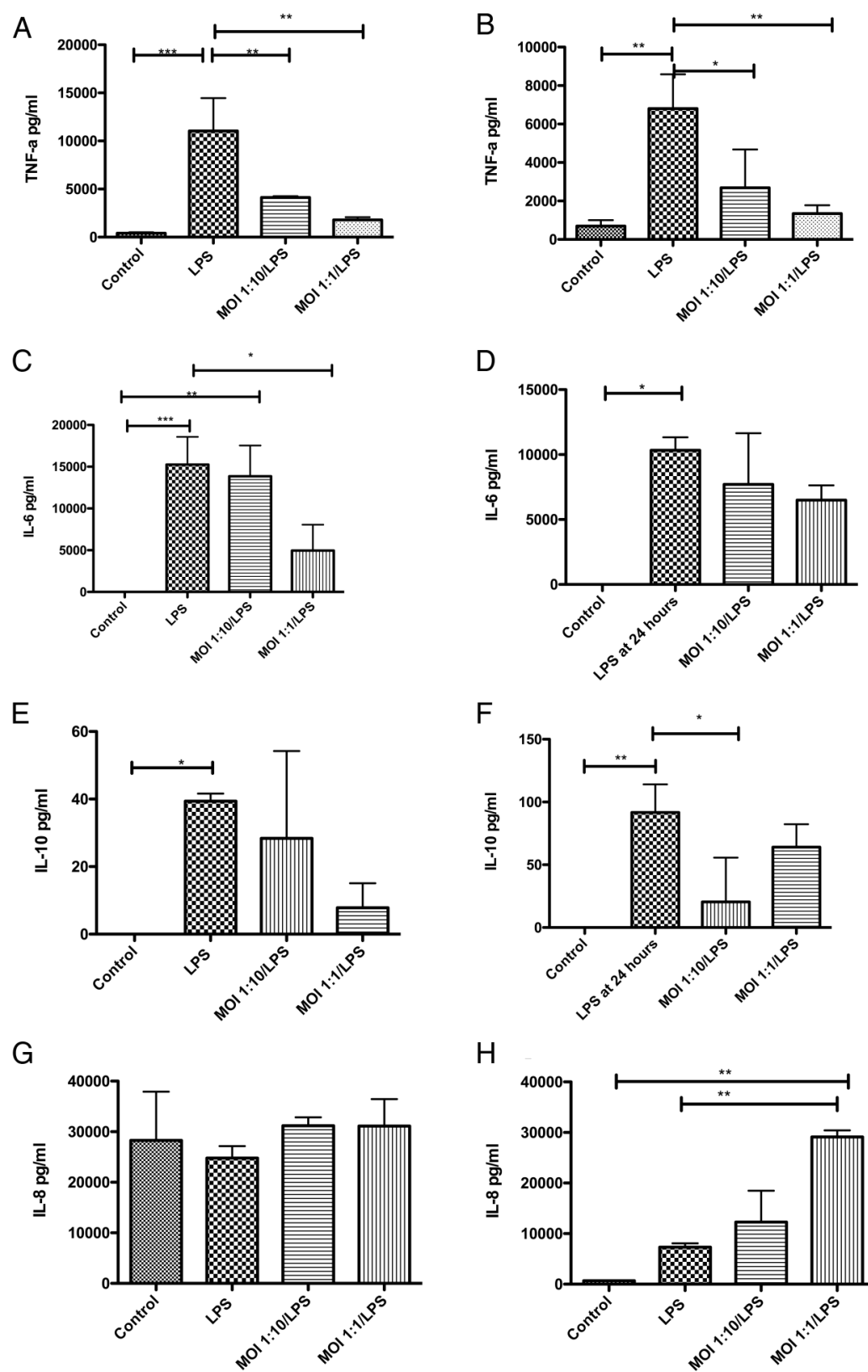

moderate titre having a more pronounced effect than low titre $(p=0.005$ by ANOVA).

\section{DISCUSSION}

This study is, to our knowledge, the most comprehensive description of Mycoplasmataceae in VAP to date. Through the use of molecular diagnostics we have confirmed previous findings of Mycoplasma presence ${ }^{78}$ in a more robust manner and, importantly, identified the species involved. We have also demonstrated the presence of small numbers of Ureaplasma spp., organisms more commonly found in genitourinary tract infections.

It is, perhaps, surprising that the dominant organism is an oral commensal which is commonly regarded as nonpathogenic. ${ }^{10}$ We did not find a single classically pathogenic Mycoplasma spp., which is in contrast to findings from authors in other countries. ${ }^{7}{ }^{8}$ However M. salivarium is found in the oral cavity of $97 \%$ of the healthy population, ${ }^{10}$ and therefore is available to be microaspirated past the endotracheal cuff alongside other organisms in the oropharyngeal space..$^{5}$ Although we undertook targeted PCR rather than pan-bacterial metagenomics, the appearance of Mycoplasmataceae may reflect a change in the lung microbiome, an effect that has been noted in other pulmonary infections. ${ }^{20}$ The role of lung microbiome shift in the pathogenesis and maintenance of pulmonary infection is an area of active, ongoing investigation. ${ }^{20}$

The striking difference in prevalence between patients with and without microbiologically confirmed VAP invites several possible explanations. First, the presence of Mycoplasma spp. in BAL may simply reflect the burden of microaspiration from the oropharynx. Second, the presence of Mycoplasma in BAL fluid may be indicative of a relative immune failure ${ }^{2}$ which leaves patients unable to clear these low pathogenicity organisms, with the immune failure being more pronounced in those who develop VAP. A third possibility is that Mycoplasma may contribute to the pathogenesis of VAP by directly impairing immune function, thus facilitating infection by other 'classical' VAP pathogens.

Although we cannot answer these questions definitively, and there may be contributions from all three, we undertook the monocyte/Mycoplasma experiments to look for proof of 


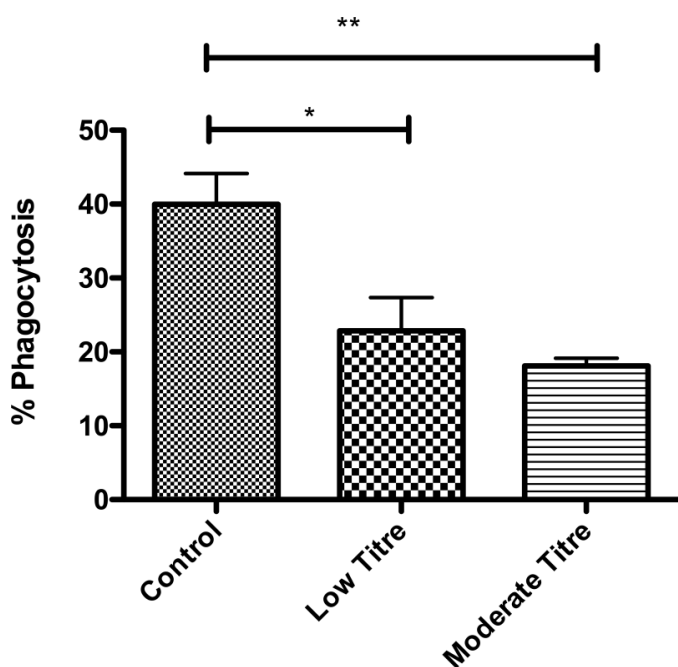

Figure 4 Phagocytosis of zymosan by monocyte-derived macrophages (MDMs) exposed to Mycoplasma salivarium. $24 \mathrm{~h}$ exposure at multiplicity of infection (MOI) of 1:10 (low) and 1:1 (moderate) titre. $p=0.005$ by ANOVA. Data shown as mean and SD. ${ }^{*} p<0.05,{ }^{* *} p<0.01$ by Bonferroni's post-hoc test, all other comparisons non-significant. Results are from duplicates of $n=4$ individual healthy donors, with 300000 cells per well, data shown as mean and $95 \% \mathrm{Cl}$.

principle of Mycoplasma-induced immune impairment that may support the third hypothesis. Among patients with HIV/AIDS, co-infection with Mycoplasma spp. is associated with faster progression of a disease which is characterised by immune failure, ${ }^{12}$ although the causative relationship remains controversial ${ }^{21} 22$ and the Mycoplasma spp. implicated are those more commonly associated with the genitourinary tract. ${ }^{21}$ In previous in vitro studies in human monocytic cell lines ${ }^{23}$ and bovine monocytes ${ }^{24}$ the presence of unspecified Mycoplasma spp. ${ }^{23}$ and M. bovis ${ }^{24}$ respectively have been associated with impaired immune responses. The mechanism by which $M$. salivarium alters innate immune cell function is the focus of ongoing work, however our data do not support this being simply an effect of cellular toxicity. Interestingly, the effects on cytokine secretion are complex with reductions in LPS-induced secretion of TNF- $\alpha$, IL- 6 and IL-10 but unaffected (monocyte) or stimulated (MDM) IL-8 production. The standard description of 'deactivated/reprogrammed' monocytes in patients with sepsis is of reduced TNF- $\alpha$ and enhanced IL-10 secretion. ${ }^{25}$ Thus the effects we have seen with Mycoplasma may reflect a related but different phenomenon to classical 'LPS tolerance'. ${ }^{25}$ Indeed Zakharova et al found a similar Mycoplasma-mediated suppression of IL-10 transcription in monocyte-like THP-1 cells. ${ }^{23}$ Although we can only speculate on the potential in vivo effects, it is possible that when Mycoplasma spp. enter the already inflamed lung their predominant suppressive effect is maladaptive and promotes infection but the same effect in its 'normal' environment contributes to an adaptive microbiota-induced immune tolerance. $^{2026}$

We believe this study has a number of strengths. First we examined a large number of samples taken from the alveolar region of the lungs of patients with suspected VAP and these samples came from a range of locations throughout the UK. It is therefore unlikely that these findings are specific to a single unit or geographical region. Second, by using the latest molecular techniques we were able (A) to identify Mycoplasma with greater confidence than previous studies which relied on
Mycoplasma toxin detection ${ }^{7}$ or limited $\mathrm{PCR}^{8},{ }^{8}$ and (B) to identify the specific species involved. Third, by undertaking examination of the effects of the most common identified species, $M$. salivarium, on two key innate immune cells, we were able to strengthen our hypothesis that this organism is not simply a bystander but is contributing to the immunoparesis that facilitates VAP.

Our study is limited by having a sample from a single time point, at the time of suspected infection. Therefore we cannot comment on when the Mycoplasmataceae appeared in the alveolar space relative to the development of VAP. As such we are certainly unable to infer a causative relationship between Mycoplasmataceae and subsequent VAP. Our positive event rate of 21 patients with Mycoplasmataceae and VAP prevents more in-depth examination of the relationship between Mycoplasmataceae, clinical features and outcomes from VAP. We were also unable to precisely quantify the amounts of non-classical organisms present in the lungs of patients, although the ability to obtain good speciation data for the great majority of the Mycoplasma positive samples from patients with VAP suggests a reasonable bacterial load. The titres of $M$. salivarium to which the monocytic cells were exposed therefore remain an approximation of the situation in vivo. Indeed our in vitro model of Mycoplasma infection is necessarily a simplification of the in vivo situation.

The implications of this work are that preventing lower respiratory tract Mycoplasma spp. infection, through improved oral hygiene measures and prevention of microaspiration, may contribute to preventing VAP through immune restoration. This hypothesis could also provide a novel explanation for the finding that incorporating macrolides to antibiotics for VAP improves outcomes, ${ }^{27}$ beyond the more widely accepted immunomodulatory effects of macrolides. ${ }^{28}$ We do not, however, advocate broadening antibiotic coverage on the basis of our findings, as only further clinical trials could justify such an approach. The high prevalence of Mycoplasmataceae among patients with VAP should also be considered when pan-bacterial molecular diagnostics, such as $16 \mathrm{~S}$ rRNA gene PCR, are being used for pathogen detection in VAP; mixed sequences are difficult to resolve using conventional Sanger-based sequencing and may require next-generation sequencing methodologies.

In conclusion we have found a high prevalence of nonclassical bacterial species among patients with VAP, specifically organisms such as $M$. salivarium that are traditionally thought to be non-pathogenic. We have further demonstrated that these organisms can alter antibacterial functions in healthy volunteer monocytes and MDMs, and may have the potential to contribute to the immunoparesis seen in critically ill patients.

Acknowledgements The authors thank the VAP-RAPID collaborators, Daniel $F$ McAuley, Niall H Anderson, Suveer Singh, Paul Dark, Alistair I Roy, Simon V Baudouin, Stephen E Wright, Gavin D Perkins, Melinda Jeffels, Cecilia M O'Kane, Craig Spencer, Shondipon Laha, Nicole Robin, Savita Gossain, Kate Gould, Marie-Hélène Ruchaud-Sparagano, Jonathan Scott, Ian Dimmick, Ian F Laurenson, Helen Walsh, Sarah Nutbrown, Charley Higham, Teresa Melody, Keith Couper, Jacqueline Baldwin, Alexandra Williams, Kylie Norrie, Julie Furneval, Tracey Evans, Heidi Dawson, Griania White, Lia McNamee, Leona Bannon, Laura Evans, Neil Young, Alasdair Hay, Ross Paterson, Stuart McLellan, Peter Kelleher, Berge Azadian, Masao Takata, Ildiko Kustos, John Cheesborough and Roland Koerne, for their work in recruiting the patients to cohort 2. The authors also thank David Swann, Pam Ramsay, Gordon McNeil and Kallirroi Kefala for their work in recruiting patients into cohort 1 . The authors also thank the patients and their relatives who allowed this research to occur.

Contributors TJN conducted the research and drafted the paper, NJG conducted the research and drafted the paper, TPH recruited patients and obtained samples and revised the paper, KET obtained funding, supervised the research and drafted the paper, RM supervised the research and revised the paper, JPM conducted the 
research and revised the paper, JR conducted the research and revised the paper, CTR conducted the research and revised the paper, TSW obtained funding, devised the study and supervised the research and revised the paper, AGR supervised the research and revised the paper, ACM obtained funding, devised the study, supervised the research and drafted the paper, AJS obtained funding, obtained samples, devised the study and drafted the paper.

Funding This study was funded by the Hospital Infection Society, Wellcome Trust/ Department of Health Health Innovation Challenge Fund (HICF)(0510/078) and Sir Jules Thorn Charitable Trust (03/JTA)

Competing interests RM is a Consultant/Advisor for Gilead Sciences Ltd and is the recipient of an Innovate UK grant with Randox Diagnostics to investigate diagnostic tests, KET is a consultant to Becton Dickinson, Cepheid, Enigma, GenMark and Selex. All other authors declare no conflicts of interest.

Provenance and peer review Not commissioned; externally peer reviewed.

Data sharing statement Additional unpublished data from this study can be obtained by direct contact with the corresponding author.

Open Access This is an Open Access article distributed in accordance with the terms of the Creative Commons Attribution (CC BY 4.0) license, which permits others to distribute, remix, adapt and build upon this work, for commercial use, provided the original work is properly cited. See: http://creativecommons.org/ licenses/by/4.0/

\section{REFERENCES}

1 Hunter JD. Ventilator associated pneumonia. BMJ 2012;344:e3325.

2 Conway Morris A, Kefala K, Wilkinson TS, et al. C5a mediates peripheral blood neutrophil dysfunction in critically ill patients. Am J Respir Crit Care Med 2009;180:19-28.

3 Morris AC, Brittan M, Wilkinson TS, et al. C5a-mediated neutrophil dysfunction is RhoA-dependent and predicts infection in critically ill patients. Blood 2011;117:5178-88.

4 Conway Morris A, Anderson N, Brittan M, et al. Combined dysfunctions of immune cells predict nosocomial infection in critically ill patients. $\mathrm{Br} \mathrm{Jn}$ Anaesth 2013;111:778-87.

5 Chastre J, Fagon JY. Ventilator-associated pneumonia. Am J Respir Crit Care Med 2002;165:867-903.

6 David LA, Maurice CF, Carmody RN, et al. Diet rapidly and reproducibly alters the human gut microbiome. Nature 2014;505:559-63.

7 Muir MT, Cohn SM, Louden C, et al. Novel toxin assays implicate Mycoplasma pneumoniae in prolonged ventilator course and hypoxemia. Chest 2011;139:305-10.

8 Mokhless NAS, El-Mofty MF, Hanafi NF, et al. Atypical bacteria in ventilator associated pneumonia; an Egyptian University Hospital experience. J Am Sci 2010;6:1074-9.

9 Razin S, Yogev D, Naot Y. Molecular biology and pathogenicity of mycoplasmas. Microbiol Mol Biol Rev 1998;62:1094-156.

10 Watanabe T, Matsuura M, Seto K. Enumeration, isolation, and species identification of mycoplasmas in saliva sampled from the normal and pathological human oral cavity and antibody response to an oral mycoplasma (Mycoplasma salivarium). J Clin Microbiol 1986;23:1034-8.

11 Baracaldo R, Foltzer M, Patel R, et al. Empyema caused by Mycoplasma salivarium. J Clin Microbiol 2012;50:1805-6.

12 Chattin-Kacouris BR, Ishihara K, Miura T, et al. Heat shock protein of Mycoplasma salivarium and Mycoplasma orale strains isolated from HIV-seropositive patients. Bull Tokyo Dent Coll 2002;43:231-6.

13 Conway Morris A, Kefala K, Wilkinson TS, et al. Diagnostic importance of pulmonary interleukin-1 beta and interleukin-8 in ventilator-associated pneumonia. Thorax 2010;65:201-7.

14 Hellyer TP, Conway Morris A, McAuley DF, et al. Diagnostic accuracy of pulmonary host inflammatory mediators in the exclusion of ventilator-acquired pneumonia. Thorax 2015:70:41-7.

15 Van Kuppeveld FJ, Van der Logt JT, Angulo AF, et al. Genus- and species-specific identification of mycoplasmas by $16 \mathrm{~S}$ rRNA amplification. Appl Environ Microbiol 1992;58:2606-15.

16 Kong F, Ma Z, James G, et al. Species identification and subtyping of Ureaplasma parvum and Ureaplasma urealyticum using PCR-based assays. I Clin Microbiol 2000;38:1175-9.

17 Haslett C, Guthrie LA, Kopaniak MM, et al. Modulation of multiple neutrophil functions by preparative methods or trace concentrations of bacterial lipopolysaccharide. Am J Pathol 1985;119:101-10.

18 Michlewska S, Dransfield I, Megson IL, et al. Macrophage phagocytosis of apoptotic neutrophils is critically regulated by the opposing actions of pro-inflammatory and anti-inflammatory agents: key role for TNF-alpha. FASEB J 2009;23:844-54.

19 Gordon SB, Read RC. Macrophage defences against respiratory tract infections. The immunology of childhood respiratory infections. Br Med Bull 2002;61:45-61.

20 Dickson RP, Erb-Downward JR, Huffnagle GB. The role of the bacterial microbiome in lung disease. Expert Rev Respir Med 2013;7:245-57.

21 Napierala Mavedzenge S, Weiss HA. Association of Mycoplasma genitalium and HIV infection: a systematic review and meta-analysis. AIDS 2009;23:611-20.

22 Taylor-Robinson D. Infections due to species of Mycoplasma and Ureaplasma: an update. Clin Infect Diseases 1996;23:671-82.

23 Zakharova E, Grandhi J, Wewers MD, et al. Mycoplasma Suppression of THP-1 cell TLR responses is corrected with antibiotics. PLOS ONE 2010;5:e9900.

24 Mulongo M, Prysliak T, Scruten E, et al. In vitro infection of bovine monocytes with Mycoplasma bovis delays apoptosis and suppresses production of gamma interferon and tumor necrosis factor alpha but not interleukin-10. Infect Immun 2014;82:62-71.

25 Cavaillon JM, Adib-Conquy M. Bench-to-bedside review: endotoxin tolerance as a model of leukocyte reprogramming in sepsis. Crit Care 2006;10:233.

26 Nakata $\mathrm{K}$, Yamamoto $\mathrm{M}$, Inagawa $\mathrm{H}$, et al. Effects of interactions between intestinal microbiota and intestinal macrophages on health. Anticancer Res 2013;33:2849-53.

27 Giamarellos-Bourboulis EJ, Pechère JC, Routsi C, et al. Effect of clarithromycin in patients with sepsis and ventilator-associated pneumonia. Clin Infect Dis 2008:46:1157-64.

28 Morikawa K, Oseko F, Morikawa S, et al. Immunomodulatory effects of three macrolides, midecamycin acetate, josamycin, and clarithromycin, on human T-lymphocyte function in vitro. Antimicrob Agents Chemother 1994;38:2643-7. 